Invited chapter for: Magnetoencephalography: From Signals to Dynamic Cortical Networks. Eds. Selma Supek \& Cheryl J. Aine, Springer Verlag, 2013

\title{
13 MEG/EEG Data Analysis Using EEGLAB
}

\author{
John R. Iversen*, Scott Makeig \\ Swartz Center for Computational Neuroscience, Institute for Neural Computation, University \\ of California San Diego. La Jolla, CA 92093-0559. \\ *Corresponding author: jiversen@ucsd.edu.
}

\begin{abstract}
EEGLAB (sccn.ucsc.edu/eeglab) is an easily extensible, highly evolved, and now widely used open source environment for signal processing of electroencephalographic data running on MATLAB (The Mathworks, Inc.). Here we introduce MEEG, an EEGLAB plug-in that appears in the EEGLAB menu of users who download it. MEEG gathers functions from EEGLAB and other MATLAB-based open source frameworks to read/write and process MEG or simultaneous MEG/EEG (MEEG) data. Here we show a first decomposition by independent component analysis (ICA) of an MEEG data set and use MEEG plotting tools to localize and evaluate maximally independent joint MEG/EEG component processes in the data.
\end{abstract}

Keywords: MEG, EEG, MEEG, Independent Component Analysis, ICA, EEGLAB, localization, radial, tangential, dipole, AMICA

\section{Introduction}

EEGLAB (sccn.ucsd.edu/eeglab) (Delorme \& Makeig 2004) evolved from an ICA Toolbox for Electrophysiological Data Analysis released by Makeig and colleagues at The Salk Institute (La Jolla CA) in 1997. Currently EEGLAB is a mature, actively evolving open-source software environment for electrophysiological data analysis running on MATLAB (The Mathworks, Inc.) that makes freely available a range of state-of-the-art approaches to describe brain dynamics of effective cortical and non-brain EEG sources at both the individual and group levels (Delorme \& Makeig 2004; Makeig et al. 2004). By a 2011 survey (Hanke \& Halchenko 2011), EEGLAB may currently be the most widely used open-source toolbox for EEG analysis. EEGLAB functions comprise a broad core range of 
functionality accessible either through its graphic user interface (GUI) and/or directly from the MATLAB command line, plus plug-in tools and toolboxes that implement a wide range of advanced analysis and visualization methods.

User interface. EEGLAB can be controlled through its GUI (Fig. 1 lower left, panel), or more directly through MATLAB scripts and command line calls. Use of the GUI is highly convenient for data exploration. The GUI also accumulates a history of the commands to EEGLAB functions it issues, enabling processing pipelines developed using the GUI to be easily turned into a MATLAB script. Already many students (worldwide) have learned to write MATLAB data analysis scripts by combining the EEGLAB history mechanism with the extensive EEGLAB function and wiki documentation (sccn.ucsd.edu/wiki/eeglab).

Other tools. EEGLAB is the center of a growing ecosystem of open source software tools (Fig. 1) that have been released by researchers at the Swartz Center for Computational Neuroscience at UCSD (sccn.ucsd.edu). These include the Human Electrophysiology, Anatomic Data, and Integrated tools (HeadIT) data archive and resource (headit.org), with its system for tagging uploaded studies (Experimental Study Schema (ESS) (Bigdely-Shamlo, Kreutz-Delgado, Kothe, et al. 2013a), Hierarchical Events Descriptors (HED) (Bigdely-Shamlo, KreutzDelgado, Robbins, et al. 2013b) and a cross-platform system for synchronized collection of data from EEG and many other devices (Lab Streaming Layer, LSL; code.google.com/p/labstreaminglayer) plus an extensible, XML-based data format (Extensible Data format, XDF; code.google.com/p/XDF) and a Python-language scripting framework for controlling simple or very complex experimental paradigms (SNAP).

MoBILAB. An object-oriented environment for analysis of multimodal data collected under the mobile brain/body imaging (MoBI) paradigm, MoBILAB (sccn.ucsd.edu/wiki/Mobilab_software) can export EEG data to EEGLAB for further analysis, and may in the future become our primary platform for developing and sharing multimodal data analysis methods, since the EEGLAB EEG data structure has limited support for different channel types and assumes all data to be recorded at the same sampling rate. For MEG/EEG data recorded at the same rate this is not much of an inconvenience, as EEGLAB provides a channel type variable that allows functions to perform EEG analysis and/or MEG analysis of the respective data channel subsets based on their specified channel types.

EEGLAB plug-ins. The growing range of EEGLAB plug-ins have been previously described (Delorme et al. 2011). Plug-ins released by SCCN itself include advanced Adaptive Mixture ICA (AMICA) for identification of maximally independent brain sources with artifact rejection (Palmer 2006; Delorme et al. 2012), the DIPFIT toolbox implementing source dipole fitting tools by Robert Oostenveld from Fieldtrip (fieldtrip.fcdonders.nl), the Neuroelectromagnetic Forward Head Modeling Toolbox (NFT) for creating detailed boundary element model (BEM) or finite element model (FEM) head models (Akalin Acar \& Makeig 2010), the Measure Projection Toolbox (MPT) for cross-subject source-level analysis using measure projection (Bigdely-Shamlo, Mullen, et al. 2013c), the Source Information Flow Toolbox (SIFT) for calculation and visualization of multivariate 
causal source dynamics in both event-related and continuous data (Delorme et al. 2011), and BCILAB, a complete toolbox for building, running, and statistically evaluating brain-computer interface (BCI) models (Kothe \& Makeig 2010). At least 20 other plug-in tools and toolboxes have been released by other research groups; these are listed on a wiki page (sccn.ucsd.edu/wiki/EEGLAB_Plugins). A facility for automated updating of listed plug-ins to new versions from within EEGLAB is planned for EEGLAB v13.

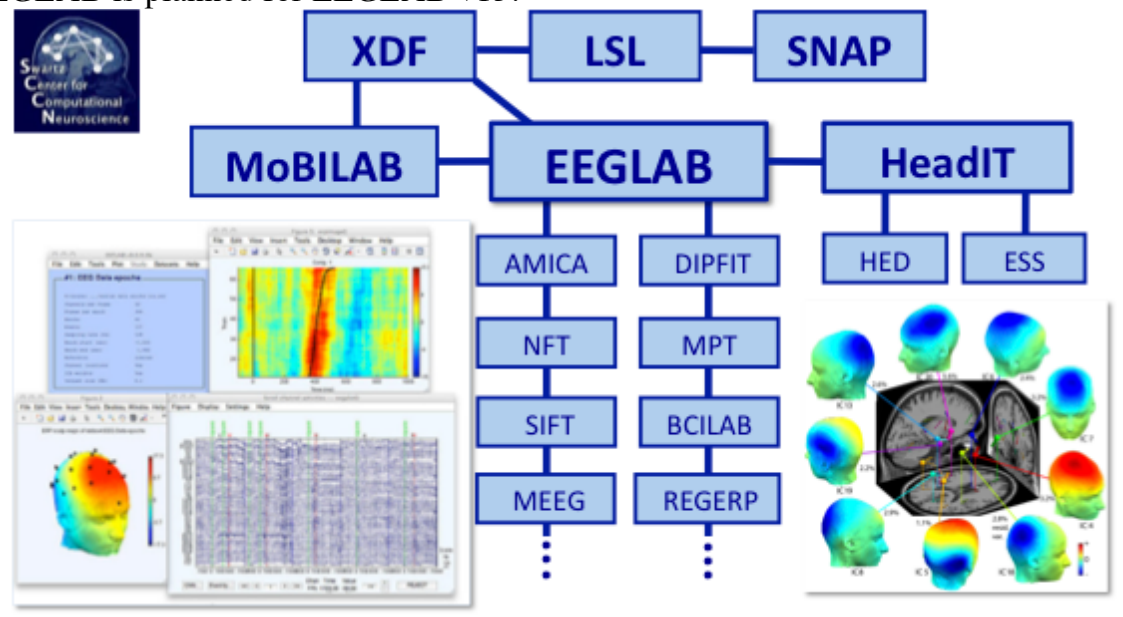

Fig. 1. The EEGLAB environment for electrophysiological signal processing is the center of a growing framework of tools developed and released by researchers at the Swartz Center for Computational Neuroscience (SCCN) at UCSD. These include software for synchronized multimodal recording (SNAP, LSL, XDF), MoBILAB, an object-oriented toolbox for analysis and visualization of multimodal data, the HeadIT data and tools resource with its associated tools (HED, ESS, etc.), and a growing set of toolboxes that operate as EEGLAB plug-ins (AMICA, DIPFIT, NFT, MPT, SIFT, BCILAB, etc.). MEEG is a new plug-in developed by the authors for analysis of MEG and MEEG (synchronized MEG plus EEG) data.

The MEEG plug-in. EEGLAB now includes an MEEG plug-in (sccn.ucsd.edu/wiki/MEEG) that expands the ability of EEGLAB users to import and analyze MEG and dual-modality MEEG (concurrent MEG and EEG) datasets and thereby opening a range of novel data analysis techniques for use by the MEG community. MEEG data handling within EEGLAB is tightly coupled to Fieldtrip, allowing the EEGLAB data structures it handles to be readily imported from and exported to Fieldtrip. Both the EEGLAB environment and the MEEG plug-ins are ongoing efforts that we hope other MEG users and methods developers will contribute to. The MEEG developers remain open to partnering with other methods developers to share capabilities between MEEG and other MEG toolboxes).

Data and experiment types supported. In addition to standard EEG data types, EEGLAB now supports the loading of MEG and MEEG data through its integration of the Fieldtrip fileio module. Individual data files can be imported as individual EEGLAB data sets, or multiple runs can be combined into a single dataset using realignment to a common sensor orientation. In addition, the new 
MEEG plug-in enables EEGLAB to import and export a range of Fieldtrip data structures, including raw and epoched data, as well as independent component analyses, so that EEGLAB processing can begin after partial analysis in Fieldtrip, or can be exported, allowing Fieldtrip to be used for additional processing. EEG recording systems provide a single scalar value per sensor location, in contrast to the wider variety of MEG sensor types. The scalar model easily accommodates magnetometer and radial gradiometer systems, but requires either magnetometers or the magnitude of the planar gradient to be chosen (e.g., for Yokogawa system data sets).

Source localization. ICA decomposition enables the profitable use of dipolebased inverse methods because of the characteristic resemblance of many MEG, EEG, or also MEEG independent component scalp maps to the projection of a single equivalent dipole, allowing them to be well-fit by a single equivalent dipole model (or, in some cases, to a dual-dipole model with symmetric location constraints) (Delorme et al. 2012). The DIPFIT toolbox in EEGLAB implements equivalent dipole model fitting tools by Robert Oostenveld from Fieldtrip (fieldtrip.fcdonders.nl). Dipole fitting tools have been integrated in the the Neuroelectromagnetic Forward head modeling Toolbox (NFT) (Akalin Acar \& Makeig 2010). These plus some novel distributed source localization methods will be put into a toolbox paralleling NFT, to be called the Neuroelectromagnetic Inverse Source modeling Toolbox (NIST).

Processing data from multiple subjects or sessions. EEGLAB supports across-subject analysis via a STUDY structure that points to a set of similar EEG datasets forming an experimental study. Currently, these datasets are typically epoched datasets (sets of data epochs similarly time locked to one or more sets of experimental events). EEGLAB Study software can prepare and store a userspecified set of continuous (power spectrum) and event-related (ERP, ERSP, ITC, etc.) measures for each dataset and help the user to separate these measures into conditions, sessions, and/or subject groups. Typically, each dataset is associated with an ICA decomposition and a list of 'brain' components to study, each with an equivalent dipole model. The Study functions can then prepare a pair-wise distance measure between components based on component dipole (and/or scalp map) and specified measure distances. Users then can cluster the components using at least three clustering methods, and can compute statistical contrasts across subjects/sessions using either parametric (Gaussian) or non-parametric (bootstrap) statistical methods. Clustering scalp channel signals, though less advised, is also supported.

Currently, users can create and process one or more $1 \mathrm{xN}$ or NxM statistical designs for a given Study. Thus, for example, given 5 different event-related measures for each subject in an experiment, the user can specify Conditions 1-4 as forming a 2x2 design, and/or can also compare Conditions 2 vs. 5 in another design, without needing to duplicate the STUDY structure and its associated measure files. Both within-subject and across-subject variable types are supported.

As in practice, the range of experimental designs is much wider (than $\mathrm{NxM}$ ), EEGLAB and some EEGLAB toolbox developers are now working with Cyril 
Pernet of the University of Glasgow to incorporate his LIMO toolbox into the core of EEGLAB study processing. It supports parametric and non-parametric statistics for a much wider range of designs (gforge.dcn.ed.ac.uk/gf/project/limo_eeg) (Pernet et al. 2011).

Measure projection. An alternate approach to component clustering is taken in the Measure Projection Toolbox (MPT) of Nima Bigdely-Shamlo (BigdelyShamlo, Mullen, et al. 2013c). This toolbox focuses on comparing component source dynamics for a single measure at a time (for example, ERPs) based on the location of the equivalent source dipole in a template brain. Each component dipole location is replaced by a 3-D Gaussian blur (representing location probability) and, after populating the template brain with source dipoles across a potentially large number of subjects, two operations are applied voxel-wise (that is, template brain voxel-by-voxel). First, brain regions in which local dipole measures agree are identified, forming a measure consistency subspace. Next, voxels in this subspace are clustered using affinity clustering to form voxel domains with distinct measure time courses. Here the concept of measure domains in the template brain volume replaces the discrete component clusters produced by the default EEGLAB study processing. Users may choose either or both paths to use to characterize their study data.

CSA clustering. Arthur Tsai of Academica Sinica, Taiwan, has recently developed an advanced approach to study source clustering (Tsai et al. 2013). This applies spatiotemporal ICA decomposition using EMSICA (Tsai et al. 2006) to EEG (or as readily, MEG) data from its projection back onto to the oriented subject cortex, modeled from a subject MR head image. The cortical surface models are then inflated and co-registered using tools available in Freesurfer (Fischl et al. 1999). Finally, source clustering across subjects is performed in the 2-D cortical surfacealigned space rather than in 3-D template brain space (as in MPT and EEGLAB Study functions). A CSA (Cortical Surface Alignment) EEGLAB plug-in is envisaged that will allow users to perform this potentially more accurate analysis when MR head images are available for the individual subjects in an EEG or MEG study.

\section{MEEG data decomposition: An empirical data example}

For example purposes, we will illustrate the capabilities of the MEEG plug-in and other EEGLAB features using a simultaneously recorded multimodal (MEEG) MEG plus EEG dataset (Bledowski et al. 2012) that is jointly decomposed, in a single AMICA decomposition, to extract independent components accounting for both MEG and EEG data streams. The validity of the decomposition is based on the assumed linearity of the underlying electric and magnetic components of the electromagnetic field generated by the effective generators of the scalp-recorded (EEG) potentials and (MEG) flux. We use the NFT toolbox to create an EEG and MEG head model and use it to fit equivalent dipole models to the resulting inde- 
pendent component (IC) scalp maps. We focus here on describing the relations between MEG signal and EEG signal projections of the resulting ICs, including a first statistical examination using ICA of the degree to which radial EEG sources (as determined by an equivalent dipole model) are also visible in MEG.

Data loading and preprocessing. The epoched CTF dataset included time series data from 269 radial gradiometers $\left(3^{\text {rd }}\right.$-order synthetic) plus 56 EEG channels. Five separate runs from the same recording session were imported and merged into a single EEGLAB dataset of size 325 channels by 580k time points. The MEEG toolbox enabled the selection of alignment across runs of the MEG data (e.g., projection onto the average across-run gradiometer locations using Fieldtrip $f t$ megrealign) as well as the choice (when appropriate) of synthetic gradiometer order. Field contributions from external sources were removed by computation of third order gradients using contributions from reference sensors (Fife 1999). The resulting EEGLAB dataset included 324 channels and 136 6-s data epochs. These data were down sampled from $1200 \mathrm{~Hz}$ to $600 \mathrm{~Hz}$, and the EEG channels were average referenced. One EEG channel was dropped following these procedures to keep the data full rank.

Artifact detection and rejection. A range of artifact rejection options are available in EEGLAB, both automated and interactive data rejection or cleaning, as well as ICA-based artifact rejection. For the dataset used here, epochs containing large artifacts had been rejected based on visual inspection.

Independent Component Analysis. The MEEG data were analyzed using AMICA to find independent components across the modalities. ICA in general proceeds from the observation that the signal measured at any sensor is a linear mixture of multiple sources within the brain (Makeig et al. 1996). The goal of the algorithm is to learn an unmixing matrix across all channels that results in a complete decomposition of the data into maximally independent components (ICs). In single-modality MEG or EEG data, many ICs have dipolar patterns of projection onto the sensors (Delorme et al. 2012). In MEEG data decompositions, both the associated MEG and EEG scalp projection maps in clearly defined components may be dipolar. In such cases, the maps are near-orthogonal and the implied equivalent dipole locations and orientations near-identical (Liu et al. 1998), showing that ICA has identified the joint electromagnetic field associated with a single source process that may be located using its well-defined MEG and EEG projection patterns also returned by ICA. The AMICA (Adaptive Mixture ICA; Palmer et al. 2007; sccn.ucsd.edu/ jason/amica_web.html) algorithm used here is the blind source separation method that performed best in a recent comparative test of 22 linear decomposition algorithms -- by both producing the greatest reduction of the strong mutual information present in the channel data, and by finding the largest number of component processes with 'dipolar' scalp maps compatible with the projection of a single cortical area or patch (Delorme et al. 2012).

The joint analysis of MEG and EEG data using independent component analysis is novel; to our knowledge it has not been previously reported. ICA itself, as a purely statistical method, has no notion of the type of signal it is decomposing or of the types of signal sources contributing independent information to the recorded 
source mixtures. Thus, to perform ICA decomposition of MEEG data, the MEG and EEG channel signals are simply concatenated into a dataset (here of 324 channels). The MEG and EEG portions of the data were individually sphered (a standard procedure to remove correlations and scale from data) before decomposition (P. A. Tukey \& J. W. Tukey 1981). Sphering serves both to make the MEG and EEG signals numerically identical in size (avoiding $\mu \mathrm{V}$ versus fT scaling issues), and to remove correlations between sensors (a standard step prior to ICA that speeds the convergence of the algorithm). The result of the joint decomposition is a collection of maximally independent components, each with a pair of spatial topographies (scalp maps) representing the spatial projections of the source onto the MEG and EEG sensors, respectively, and a joint MEG/EEG time course of activation across the trials.

Forward and inverse source modeling. The NFT toolbox was used to warp an MNI template 4-layer BEM model to the individual head shape defined by the EEG electrode locations. The EEG head model used the full BEM model, with forward solutions solved with METU-BEM (Akalin Acar \& Gençer 2004). The MEG head model used the inner mesh of the BEM model (inner skull surface) to define a single-shell BEM model (Hämäläinen \& Sarvas 1989). When individual anatomical MRIs are available, the NFT toolbox can use them to segment and create individual electrical and magnetic forward head models. NFT also generates lead field matrices for 3-D grid (FEM) source space or for a cortically constrained (BEM) source-space, e.g. constructed using the Freesurfer toolbox (surfer.nmr.mgh.harvard.edu). The head models and lead fields generated by the NFT toolkit can likewise be used for volumetric or cortically constrained inverse solutions in other data analysis packages. Dipoles were fit to all components automatically, with a separate dipole fit for the MEG and EEG IC topography. Each fit was characterized by its residual variance, as well as its direction with respect to the radial direction (as defined in relation to a best-fit sphere, fit to the scalp surface).

\section{Results: ICA analysis of MEEG data}

Fig. 2 shows 'ERP image' plots of trial-by-trial activities of four functionally distinct ICs from this data set. Each panel shows the IC topography for EEG and MEG in the upper left. The EEGLAB erpimage function produces a raster image generated by stacking event-related trials (in any specified order) as horizontal colored lines, where color represents signal value. Consistent evoked response activity across trials time locked to events with consistent trial latencies appears as vertical bands of color. Smoothing (vertically) lightly across trials can highlight these regularities. Here, the dashed black lines show the onset of visual stimulus presentations, and the trials are sorted in order of increasing participant reaction time to the cue stimulus (the curving black trace indicating the moment of the button press in each trial). 
In Fig. 2, evoked responses of four components demonstrate ICAs tendency to isolate functionally distinct brain responses from the recorded mixture, and that this naturally generalizes to multimodal recordings. A visual cortex IC (a) follows onsets of visual stimuli. Note the associated dipolar and near-orthogonal MEG and EEG scalp maps. The evoked response of a somatomotor cortex IC (b) is primarily time locked to (before and after) participant button presses, and again has nearorthogonal MEG and EEG scalp maps. A right frontal-cortex IC (c), whose spectrum had a broad peak in the theta band (not shown), produces increased theta band power (not shown here) during presentation of memorandum $\left(1^{\text {st }}\right)$ stimuli and subsequent $\left(3^{\text {rd }}\right)$ probe stimuli. Some of this theta burst energy was phase locked across trials; thus, the evoked response of this IC to the memoranda ( $1^{\text {st }}$ stimuli) resembles a theta burst superimposed on a slower ERP base. Note the near-radial scalp pattern of the EEG scalp map, and the corresponding lack of definition of the (weak) MEG IC projection (discussed further below). The ERP image plot for an IC accounting for eye blinks (d) shows that the participant blinked consistently during fixation intervals. Again, the MEG and EEG projections are well defined, consistent with sources in the eyes themselves, and are near orthogonal.

Fig. 2. (next page) Four 'ERP image' panels showing trial-by-trial activities of four MEEG independent components. The experiment trial design is depicted above panel 1): in each trial, a target array of colored squares that are to be memorized is briefly presented, then replaced by a fixation dot during a retention interval. A single colored probe square is then presented; the participant had to respond whether or not it was present in the initial color array. In each erpimage panel, vertical dashed black lines indicate the onset of each visual stimulus (heavier lines for target and probe stimuli; lighter lines for onsets of fixation dots). The large color image within each panel represents a raster image of all 136 individual trials, with IC activation coded by color. Activation units are proportional to projected rms EEG $\mu \mathrm{V}$ and MEG fT. The trials are sorted in order of descending reaction time, so the trace of button press moments (dark solid trace) forms a diagonal arc. In the erpimage panels, the trial activations have been (vertically) smoothed with a 10 -trial moving window. Below each erpimage panel is the standard trial average activation ERP. EEG and MEG IC topographies are shown in the upper left of each panel. a) A visual (occipital) IC (with clear, near-orthogonal EEG and MEG topographies) showing consistent evoked responses time-locked to presentations of visual stimuli. b) A somatomotor IC (again with clear, near-orthogonal EEG and MEG projections) whose evoked responses are time locked primarily to button presses. c) A near-radial right frontal theta band dominant component with weak and less clearly defined MEG projection. Response to target and probe stimuli can be modeled as a theta band burst superimposed on a lower-frequency response, and d) an eye blink IC (with clearly defined, near-orthogonal MEG and EEG projections; 2 trial smoothing window). Separation of the signals into maximally independent component processes separates out processes that are maximally functionally distinct as well. 

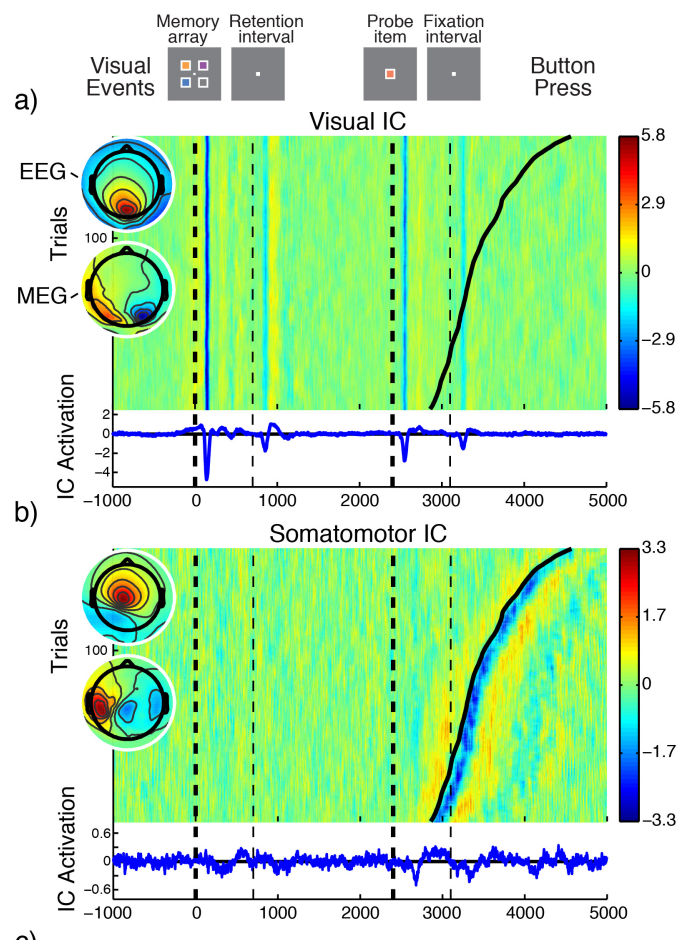

c)

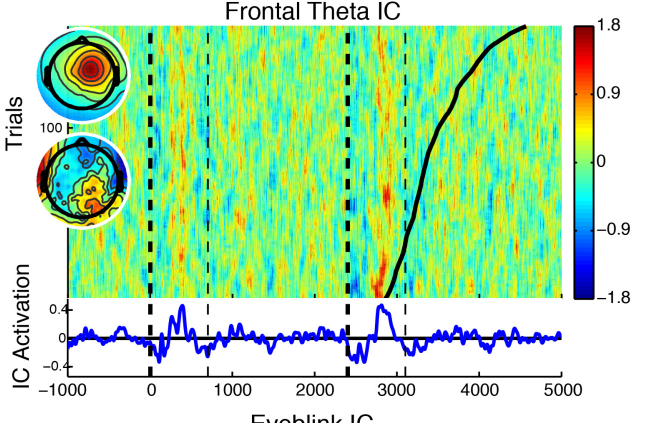

d)

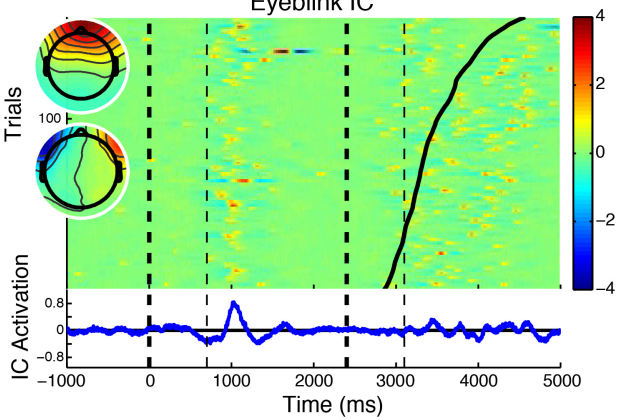


Fig. 3 shows a more complete set of IC MEG and EEG topographies for (brain and non-brain process) ICs accounting for the most signal variance among the 324 ICs returned by AMICA (pvaf = percent variance accounted for, the leftmost number above each topography). Each IC is represented as a vertical pair of head cartoons depicting the spatial projection of the IC onto the EEG (top) and MEG (bottom) sensor arrays. As usual, the ICs accounting for the most signal variance in each modality are artifactual (top row): an IC accounting for eye blinks (accounting for $12.6 \%$ of EEG signal variance), and another accounting for cardiographic contributions (in these data accounting for $21.7 \%$ of MEG signal variance). The relative sensativity of each modality to different artifact types is apparent in the pvaf values: Eye blinks and muscles account for proportionally more EEG then MEG variance, while for heart-related and line-noise artifacts the reverse holds. Many of the maps show dipole-like ('dipolar') topographies. AMICA analysis produced a pair of spatially near-orthogonal topographies for the MEG and EEG projections of the identified joint electromagnetic source processes, consistent with an origin in a single cortical patch or non-brain generator. Nonbrain components (top two rows) were so classified on the basis of having identifiable non-brain time courses (Eye \& EKG components) or a large high-frequency spectrum consistent with myographic (or line noise) activity together with equivalent dipole localized to outside the brain volume (myographic or line noise sources). Identified Brain components have equivalent dipoles (indicated in black) located within the brain volume (here with residual variance of the dipole fit $<=$ $20 \%$ ). Dipole localization is discussed further below.

Fig. 3. (next page) Results of the MEEG data joint independent component decomposition. Joint independent component (IC) topographies representing the projection patterns of individual ICs to the EEG (upper map) and MEG (lower map) sensor arrays as viewed from above the head. Each IC is represented by a vertical pair of EEG and MEG topographies. Numbers above each sensor map indicate percentage of (EEG or MEG) data variance explained (pvaf, percent variance accounted for); in brackets, the residual variance of the equivalent dipole fit to the scalp map (shown as a black dot and line on the maps), and the angle (relative to radial of a best-fit sphere) of the equivalent dipole. Depicted non-brain (top two rows of four ICs) and brain (bottom two rows) ICs are the 16 (of 324) accounting for most signal variance in each category. The non-brain component processes account for eye blinks, cardiographic sources, $(50-\mathrm{Hz})$ line noise, and scalp muscle activity, as labeled. The pair of MEG and EEG scalp maps for most components are near orthogonal, consistent with a single cortical or non-brain source. This holds for brain ICs having more tangential EEG topographies and equivalent dipoles, while (as expected) dipoles with a near-radial EEG maps and equivalent dipoles have weak (low-pvaf), and less dipolar MEG projections (i.e., single equivalent dipole model for these MEG scalp maps have higher residual variance). 

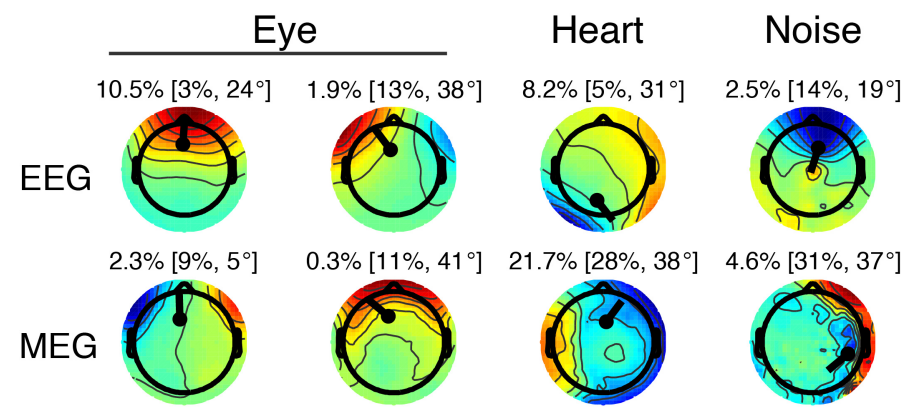

Muscle

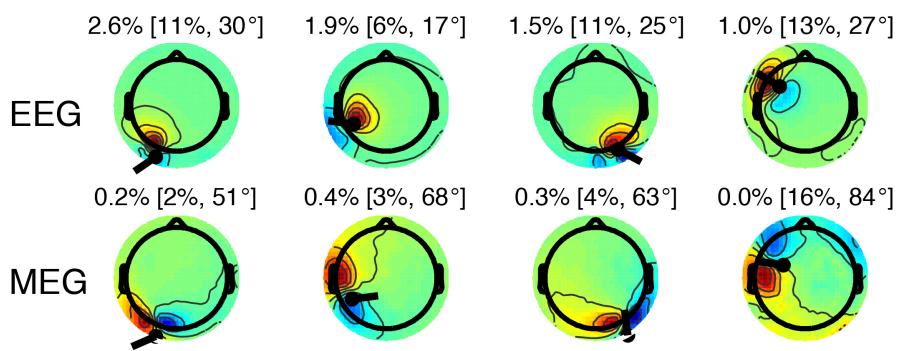

\section{Brain, Non-Radial}

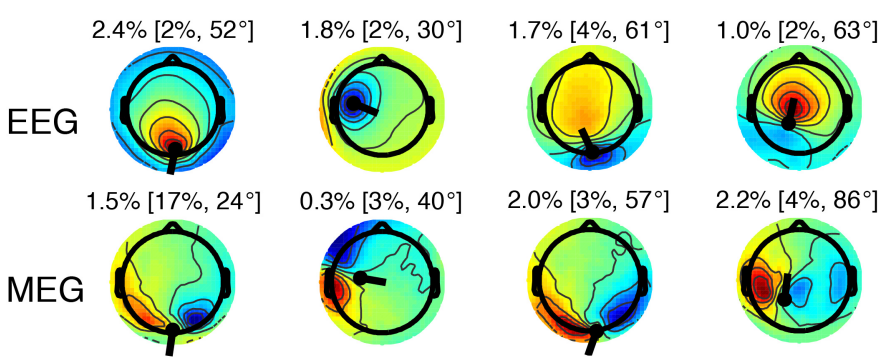

Brain, Radial

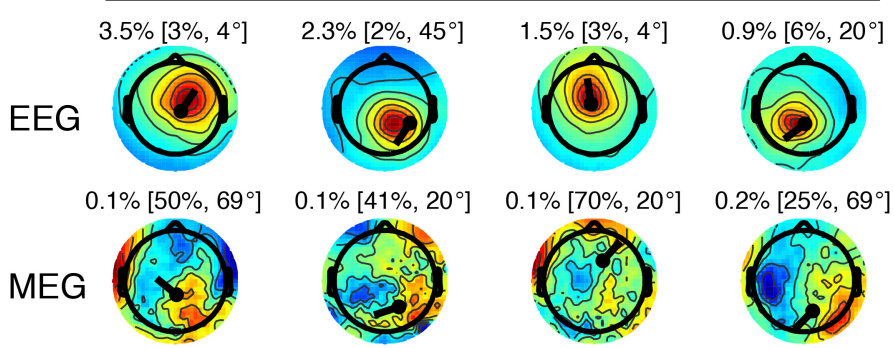


As is well known, MEG is much less sensitive to the radial component of brain current sources. In joint MEEG data ICA decompositions, this relationship falls out naturally: sources with a strong radial component have weak and usually less well-defined MEG projections. For example, the four brain components in the bottom row of Fig. 3 have large EEG projections, accounting for between $3.5 \%$ and $0.9 \%$ of total signal variance $3.5 \%$ was the largest pvaf value of any brain component). Low residual-variance dipole fits to the IC EEG scalp map return a near radial equivalent dipole (e.g., in 3 of these 4, with radial angle defined relative to a best-fit spherical head model). In contrast, the associated MEG scalp maps for these ICs have quite low pvaf $(<0.2 \%)$ and are not dipolar (residual variances, $25 \%$ to $70 \%$ ). To check for the presence of this pattern overall in the decomposition, in Fig. 4 we plot, for each dipolar, brain-based IC, the ratio of variance accounted for in the whole EEG and MEG signals (EEG pvaf / MEG pvaf) as a function of the angle from radial of the EEG equivalent dipole. Relative variance explained by the MEG portion of ICs is reduced twentyfold as the best fit dipole angle approaches a radial direction, and is close to 1:1 for tangential dipoles, in accordance with general expectations, and more specifically with expectations that the MEG component of a radial source dipole in a real head should be about $5-10 \%$ of that to a tangential source dipole (Ahlfors et al. 2010; Menninghaus \& Lütkenhöner 1995).

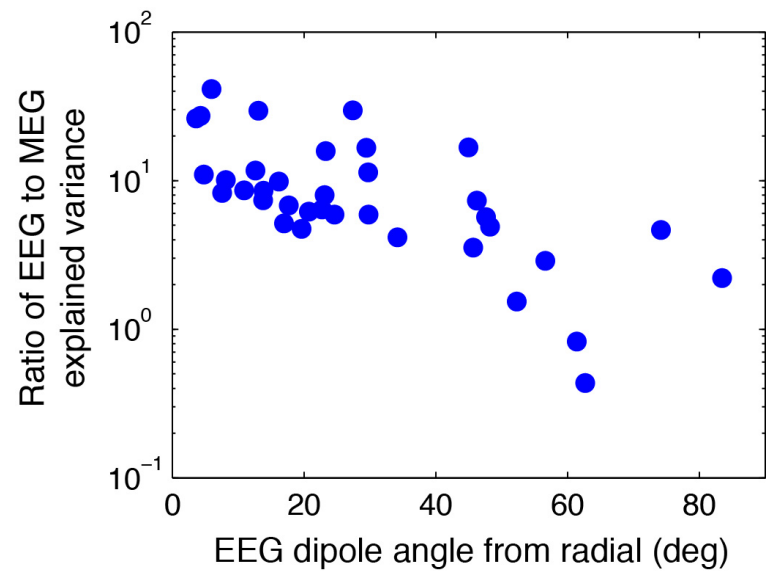

Fig. 4. Ratios of relative EEG/MEG strengths (as ratio of the percentages of MEG and EEG signal variance accounted for, on a log scale) for returned independent MEEG components with near-dipolar scalp maps (less than $20 \%$ residual variance of the single equivalent dipole model in at least one of the modalities), as a function of the deviation of the angle from radial of the EEGmap equivalent dipole. Note the expected dominance of the EEG current projections, relative to the MEG field projections, of the ICA identified near-radial sources. 


\section{Conclusions}

For EEG (Makeig et al. 1996), fMRI (McKeown et al. 1998), MEG (Ikeda \& Toyama 2000), ECoG (Whitmer et al. 2010), and other biomedical data modalities, ICA has become a widely accepted approach that provides a powerful method for identifying and separating out separate information sources in multichannel data each of whose channel signals sums activity from more than one (often, not directly recorded) source.

Here we have demonstrated that ICA may at least complement other methods for jointly analyzing simultaneously recorded EEG and MEG data (Dale \& Sereno 1993; Fuchs et al. 1998; Takada et al. 2000; Huang et al. 2007; Trujillo-Barreto et al. 2008). Its benefits may include improved source localization due to the recovery of dipole-like components with small source projections. Near-radial sources appear as those with with poorly defined MEG projections, and may be better located by inverting their simultaneously recorded and subsequently ICA-recovered electrical correlate. In addition, MEEG decomposition by ICA gives direct information on the relative scaling of MEG and EEG signals projected by cortical (and other) data sources. ICA decomposition of MEEG data should also allow principled examination of claims that MEG and EEG sources may sometimes have different spatial distributions. If and when this were the case, some class or classes of independent component processes returned by ICA applied to MEEG data should have very little EEG or MEG power. Here we showed that in our sample data set the latter was the case for EEG processes with a net radial orientation, as expected from theory.

We believe the EEGLAB environment, now augmented with the MEEG plugin incorporating several data loading and handling functions from Fieldtrip, as well as custom handling of the MEEG data within EEGLAB, is suitable for performing a range of custom MEG data analyses using available EEGLAB tools and its growing family of plug-in toolboxes. For students and researchers exploring new data sets, the EEGLAB GUI and palette of data visualization methods offers a ready way to explore data features and data quality, while its core support for data decomposition by advanced ICA methods including AMICA, and further analyses using the IC component basis, provide a powerful platform for information- and biophysics-based data modeling and statistical testing of experimental hypotheses.

Acknowledgments The authors thank Michael Wibral and colleagues for the use of the MEG/EEG data set, and Jason Palmer, Zeynep Akalin Acar, and Arnaud Delorme for useful discussions. This work was funded by a gift from The Swartz Center (Old Field NY) and from grant R01 NS047293-09 from the National Institutes of Health USA.

\section{References}

Ahlfors, S.P. et al., 2010. Sensitivity of MEG and EEG to Source Orientation. Brain topography, 
23(3), pp.227-232.

Akalin Acar, Z. \& Gençer, N.G., 2004. An advanced boundary element method (BEM) implementation for the forward problem of electromagnetic source imaging. Physics in Medicine and Biology, 49(21), pp.5011-5028.

Akalin Acar, Z. \& Makeig, S.D., 2010. Neuroelectromagnetic forward head modeling toolbox. Journal of Neuroscience Methods, 190(2), pp.258-270.

Bigdely-Shamlo, N., Kreutz-Delgado, K., Kothe, C.A., et al., 2013a. Source-Resolved EEG Measure Search. In IEEE GlobalSIP. San Antonio, TX.

Bigdely-Shamlo, N., Kreutz-Delgado, K., Robbins, K., et al., 2013b. Hierarchical Event Descriptor (HED) Tags for Analysis of Event-Related EEG Studies. In IEEE GlobalSIP. San Antonio, TX.

Bigdely-Shamlo, N., Mullen, T., et al., 2013c. Measure projection analysis: a probabilistic approach to EEG source comparison and multi-subject inference. NeuroImage, 72, pp.287303.

Bledowski, C. et al., 2012. Separable Neural Bases for Subprocesses of Recognition in Working Memory. Cerebral Cortex, 22(8), pp.1950-1958.

Dale, A.M. \& Sereno, M.I., 1993. Improved Localization of Cortical Activity by Combining EEG and MEG with MRI Cortical Surface Reconstruction: A Linear Approach. Journal of cognitive neuroscience, 5(2), pp.162-176.

Delorme, A. \& Makeig, S.D., 2004. EEGLAB: an open source toolbox for analysis of single-trial EEG dynamics including independent component analysis. Journal of Neuroscience Methods, 134(1), pp.9-21.

Delorme, A. et al., 2011. EEGLAB, SIFT, NFT, BCILAB, and ERICA: New Tools for Advanced EEG Processing. Computational Intelligence and Neuroscience, 2011, pp.1-12.

Delorme, A. et al., 2012. Independent EEG sources are dipolar. PLoS ONE, 7(2), p.e30135.

Fife, A.A., 1999. Synthetic gradiometer systems for MEG. IEEE Transactions on Applied Superconductivity, 9(2), pp.4063-4068.

Fischl, B., Sereno, M.I. \& Dale, A.M., 1999. Cortical surface-based analysis. II: Inflation, flattening, and a surface-based coordinate system. NeuroImage, 9(2), pp.195-207.

Fuchs, M. et al., 1998. Improving source reconstructions by combining bioelectric and biomagnetic data. Electroencephalography and clinical neurophysiology, 107(2), pp.93-111.

Hanke, M. \& Halchenko, Y.O., 2011. Neuroscience Runs on GNU/Linux. Frontiers in Neuroinformatics, $5, \mathrm{p} .8$.

Hämäläinen, M.S. \& Sarvas, J., 1989. Realistic conductivity geometry model of the human head for interpretation of neuromagnetic data. IEEE transactions on bio-medical engineering, 36(2), pp.165-171. 
Huang, M.-X. et al., 2007. A novel integrated MEG and EEG analysis method for dipolar sources. NeuroImage, 37(3), pp.731-748.

Ikeda, S. \& Toyama, K., 2000. Independent component analysis for noisy data--MEG data analysis. Neural networks : the official journal of the International Neural Network Society, 13(10), pp.1063-1074.

Kothe, C.A. \& Makeig, S.D., 2010. BCILAB: A BCI / EEG research framework. Proceedings, Fourth International Brain-Computer Interface Meeting.

Liu, A.K., Belliveau, J.W. \& Dale, A.M., 1998. Spatiotemporal imaging of human brain activity using functional MRI constrained magnetoencephalography data: Monte Carlo simulations. Proceedings of the National Academy of Sciences of the United States of America, 95(15), pp.8945-8950.

Makeig, S.D. et al., 2004. Mining event-related brain dynamics. Trends in Cognitive Sciences, 8(5), pp.204-210.

Makeig, S.D., Bell, A.J. \& Jung, T.-P., 1996. Independent component analysis of electroencephalographic data. Advances in neural ....

McKeown, M.J. et al., 1998. Analysis of fMRI data by blind separation into independent spatial components. Human Brain Mapping, 6(3), pp.160-188.

Menninghaus, E. \& Lütkenhöner, B., 1995. how silent are deep and radial sources in neuromagnetic measurements. In C. Baumgartner, ed. Biomagnetism: Fundamental Research and Clinical Applications. Elsevier Science, pp. 352-356.

Palmer, J.A., 2006. Variational and scale mixture representations of non-Gaussian densities for estimation in the Bayesian linear model: Sparse coding, independent component analysis, and minimum entropy segmentation. University of California, San Diego.

Palmer, J.A. et al., 2007. Modeling and estimation of dependent subspaces with non-radially symmetric and skewed densities. In Independent Component Analysis and Signal Separation. Heidelberg: Springer, pp. 97-104.

Pernet, C.R. et al., 2011. LIMO EEG: A Toolbox for Hierarchical LInear MOdeling of ElectroEncephaloGraphic Data. Computational Intelligence and Neuroscience, 2011(3), pp.1-11.

Takada, K., Nomura, K. \& Ono, H., 2000. MEG/EEG Hybrid Method for Source Localization of a Dipole with Radial Component. Papers of Technical ....

Trujillo-Barreto, N.J., Aubert-Vázquez, E. \& Penny, W.D., 2008. Bayesian M/EEG source reconstruction with spatio-temporal priors. NeuroImage, 39(1), pp.318-335.

Tsai, A.C. et al., 2013. Cortical Surface Alignment in Multi-Subject Spatiotemporal Independent EEG Source Imaging. Journal of Neurosciencce, in press, pp.1-28.

Tsai, A.C. et al., 2006. Mapping single-trial EEG records on the cortical surface through a spatiotemporal modality. NeuroImage, 32(1), pp.195-207. 
Tukey, P.A. \& Tukey, J.W., 1981. Graphical display of data sets in three or more dimensions. In V. Barnett, ed. Interpreting Multivariate Data. New York: Wiley.

Whitmer, D. et al., 2010. Utility of independent component analysis for interpretation of intracranial EEG. Frontiers in Human Neuroscience, 4, p.184. 